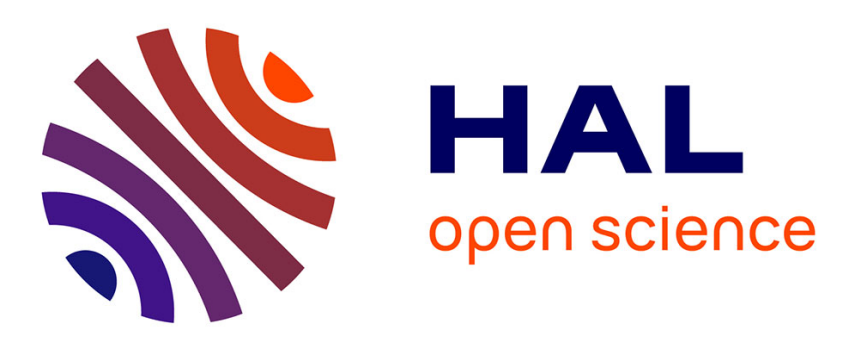

\title{
Accuracy of topological analysis of gridded electron densities
}

Philippe Rabiller, Mohamed Souhassou, Claudine Katan, Carlo Gatti, Claude Lecomte

\section{- To cite this version:}

Philippe Rabiller, Mohamed Souhassou, Claudine Katan, Carlo Gatti, Claude Lecomte. Accuracy of topological analysis of gridded electron densities. Journal of Physics and Chemistry of Solids, 2004, 65 (12), pp.1951-1955. 10.1016/j.jpcs.2004.08.006 . hal-01417558

HAL Id: hal-01417558

https://hal-univ-rennes1.archives-ouvertes.fr/hal-01417558

Submitted on 15 Dec 2016

HAL is a multi-disciplinary open access archive for the deposit and dissemination of scientific research documents, whether they are published or not. The documents may come from teaching and research institutions in France or abroad, or from public or private research centers.
L'archive ouverte pluridisciplinaire HAL, est destinée au dépôt et à la diffusion de documents scientifiques de niveau recherche, publiés ou non, émanant des établissements d'enseignement et de recherche français ou étrangers, des laboratoires publics ou privés. 


\title{
Accuracy of topological analysis of gridded electron densities
}

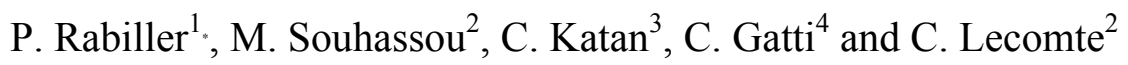 \\ 1 Groupe Matière Condensée et Matériaux, UMR-CNRS 6626, Université de Rennes 1, Campus de \\ Beaulieu, Rennes, 35042, FRANCE \\ 2 Laboratoire de Cristallographie et de Modélisation des Matériaux Minéraux et biologiques, UMR- \\ CNRS 7036, Université H. Poincaré - Nancy 1, Vandoeuvre lès Nancy, 54506, FRANCE \\ 3 Synthèse et Electro-Synthèse Organique, UMR-CNRS 6510, Université de Rennes 1, Campus de \\ Beaulieu, Rennes, 35042, FRANCE \\ 4 Istituto di Scienze e Technologie Molecolari, Milano, via Golgi, 20133, ITALY
}

\begin{abstract}
Topological analysis of electron densities sampled on 3D grids have been performed on two different crystalline compounds - ammonium dihydrogen phosphate and urea using the software package InteGriTy and the results are compared to that of analytical derivation from the software Newprop and TOPOND. Both critical points and integrated quantities are considered with emphasis put on bond critical points and atomic charges.
\end{abstract}

Keywords: D. electronic structure, C. X-ray diffraction, C. ab initio calculations, $A$. organic compounds, A. inorganic compounds.

\section{Introduction}

Topological analysis of very accurate electron densities is something quite popular nowadays $[1,2]$. During the past decade several programs have been developed. Almost all of them are either connected to a computer program [3-8] or have limitations 
concerning the type of basis sets used to describe the density [9-11]. Very recently a software package called InteGriTy [12] has been developed for the topological analysis of electron densities sampled on 3D grids. Topological analysis is based on one hand on the location and characterisation of critical points of the electron distribution and on the other hand on the unique partition of this latter according to atomic basins bounded by a zero flux surface [13]. This implies an extensive use of the density and its first (gradient) and second (hessian and laplacian) derivatives. In most of the existing programs, these quantities are derived from analytical expression of the density, which depends on the choice of basis sets. In the InteGriTy package, these quantities are obtained from a $3^{\text {rd }}$ order polynomial interpolation method applied to the density grid points [12]. This enables a rapid and systematic study over the whole grid (which can represent a crystallographic unit cell or an isolated system) that can be done without the knowledge of atomic positions. This method is particularly useful in the case of densities derived from the maximum entropy method applied to X-ray diffraction data $[14,15]$. In the study of a charge transfer complex [16] this method has been successfully applied to theoretical electron densities [17].

This paper is devoted to a comparison of the results obtained from InteGriTy and two other "analytical" softwares Newprop [5] and TOPOND [3] for two test compounds Newprop is devoted to the topological analysis of experimentally derived electron densities using a multipolar expansion [18] whereas TOPOND is dedicated to theoretical densities based on gaussian basis sets [19].

Critical points $(\mathrm{CP})$ of the electron distribution $\rho(\mathbf{r})$ correspond to positions where the gradient of the density vanishes $\nabla \rho(\mathbf{r})=\mathbf{0}$. In either InteGriTy and Newprop programs the search is based on the conventional Newton-Raphson method [20] where approaching a $\mathrm{CP}$ is realised with subsequent steps given by the product of the hessian 
matrix of the density $\mathbf{H}[\rho]$ (second derivatives) and the gradient using a damping factor $\alpha:$

$$
\mathbf{r}_{i+1}-\mathbf{r}_{\mathrm{i}}=-\alpha \cdot H \cdot \nabla \rho(\mathbf{r})
$$

The process is stopped when a limit $\varepsilon_{\mathrm{g}}$ of the gradient of the density is reached: $|\nabla \rho(\mathbf{r})|<\varepsilon_{\mathrm{g}}$. Then the curvatures $\left\{\lambda_{1}, \lambda_{2}, \lambda_{3},\right\}$ of the density at the critical points can be obtained from diagonalisation. of the hessian matrix. Each CP is characterised by two numbers $(\omega, \sigma)$ where the rank $\omega$ is the number of non zero curvatures and the signature $\sigma$ is sum of the signs of the curvatures. The laplacian $\nabla^{2} \rho(\mathbf{r})$ (trace of $\mathrm{H}$ or sum of the $\lambda^{\prime}$ s) as well as the ellipticity $\varepsilon=\left(\lambda_{1} / \lambda_{2}-1\right)$ are additional quantities that characterise a CP $[21,22]$. InteGriTy performs a systematic search throughout the whole grid starting from all the grid points [11] whereas Newprop does such a search starting from 2D grids in several planes. TOPOND searches for the different kind of CP's using a fully automated procedure [23]. In the case of $(3,-1) \mathrm{CP}$ 's, search is started from points on lines connecting two any unique pair of atoms and using either Newton-Raphson methods or two other more sophisticated alternatives [ 24, 25].

In all three programs the atomic surface $\mathrm{S}_{\mathrm{a}}$ of an atomic basin $\Omega$ is determined by single intersection of $\mathrm{S}_{\mathrm{a}}$ with rays originating from the $(3,-3) \mathrm{CP}$ to which $\Omega$ is bounded to. Integration of atomic quantities such as volume, charge and higher moments of electronic distribution are performed in spherical coordinates $(r, \theta, \varphi)$. Newprop makes integration with the same fixed numbers of integration points in $\theta$ and $\varphi$ for all the atoms. TOPOND uses such a fixed sets for each atoms. Both programs dynamically define the number of radial steps according to the length of the rays. InteGriTy adjusts all the three numbers of integration steps to predefined convergence levels according to the Romberg integration method $[12,26]$. This procedure allows for speeding the 
integration process. The major limitation of integration accuracy with InteGrity is the need to separate "valence" electron density from the total density to get reliable and precise integrated atomic charges if one cannot afford too fine grid meshes. This effect is due to the interpolation failure close to the nuclei when very sharp behaviour of the density occurs and this becomes more important as heavy atoms are considered.

\section{Experimental Density}

The first test compound $\mathrm{NH}_{4} \mathrm{H}_{2} \mathrm{PO}_{4}$ has been selected for the comparison of InteGriTy with analytical derivation of topological results from multipolar expansion with Newprop program. In this compound the ammonium $\left(\mathrm{NH}_{4}\right)$ sub-lattice is linked to the dihydrogen phosphate $\left(\mathrm{H}_{2} \mathrm{PO}_{4}\right)$ network via different kinds of intermolecular hydrogen bonds [27]. On the other hand it has received special attention [28]. In order to compute its topological properties with InteGriTy, a grid of $140 \times 140 \times 140$ points with a mesh size of $\Delta=0.05 \AA$ has been built from the multipolar refinement.

\subsection{Critical Points}

In both analytical and grid methods, a 0.3 damping factor has been used for the Newton-Raphson method. The zero gradient threshold $\varepsilon_{\mathrm{g}}$ is $10^{-8} \mathrm{e}^{-} / \AA^{4}$ for Newprop and values ranging from $10^{-8}$ to $10^{-12} \mathrm{e}^{-} / \AA^{4}$ have been used for InteGriTy. Due to the Slater type of the radial functions used in the multipolar expansion, cusp condition can appear at nucleus positions and then not all the $(3,-3)$ critical points can be accurately determined. Therefore $(3,-3)$ CP's are not systematically searched for in program Newprop. In the case of InteGriTy, $(3,-3)$ critical points can be located within less than $0.03 \AA$ from the nuclei with $\varepsilon_{\mathrm{g}}$ above $10^{-9} \mathrm{e}^{-} / \AA^{4}$. The density and curvatures show fluctuations for equivalent positions and some of them remain unrealistic (see section 3.1). Nevertheless systematic location of $(3,-3)$ critical points which correspond to 
attractors can be very useful for studying densities obtained by maximum entropy methods $[14,15]$. Then comparison is made focusing only on bond critical points (BCP) which are the more relevant for quantitative characterisation of interactions. When using InteGriTy well numerically converged CP's curvatures need a zero gradient threshold below $10^{-11} \mathrm{e}^{-} / \AA^{4}$ at the expense of CPU time and location of all $(3,-3)$ CP's. Table-1 gives the main characteristics of ammonium dihydrogen phosphate intramolecular and intermolecular hydrogen bonds $(3,-1)$ BCP's. In both cases the results obtained agree very well and there is no noticeable difference in the position and the density at the critical points.

Newprop and InteGriTy use the same algorithm to reach the precise position of CP's. They only differ in the way the density and its derivatives are obtained, that is respectively from analytical and interpolation expressions. This means that at the level employed for mesh size and Newton-Raphson method criteria, the interpolation method implemented in InteGriTy gives reliable results while the CPU time is in favour of InteGriTy (less than 3 minutes on a $1 \mathrm{GHz}$ PC computer compared to about half an hour for Newprop). Two slightly different sets of curvature values are found for intramolecular $\mathrm{O}-\mathrm{H}$ bond critical points (see $2^{\text {nd }}$ and $3^{\text {rd }}$ rows of table-1). As they should be equal to comply with symmetry, this gives thus an idea of the accuracy of the grid method for the $0.05 \AA$ mesh size used in this case.

\subsection{Integrated quantities}

The comparison between InteGriTy and Newprop for integrated atomic volumes and charges is reported on table-2. The main difference in the integration processes lies in the fact that Newprop uses gaussian quadrature method with a fixed number of integration points along theta and phi (they do not vary during the surface search). On 
the other hand, Integrity uses an iterative procedure with relative integration convergence levels, which have been set here respectively to $10^{-7}$ and $10^{-4}$ for radial and angular integration. Newprop integrated atomic charges are determined from the total electron density whereas atomic charges derived with the help of InteGriTy come from the valence electron density alone. As can be seen on table-2, the results again agree very well and CPU time consuming is in favour of InteGriTy (on the order of 5 min per atom) provided grid building time remains reasonable. The total charge error over the unit cell is less than $0.1 \%$ of the 160 valence electrons $(0.037$ electron per chemical formulae for InteGriTy against 0.047 for Newprop) whereas the added basins' volume is about $1.7 \%$ less than the unit cell volume $\left(420.5 \AA^{3}\right)$.

\section{Theoretical Density}

Urea $\mathrm{CONH}_{2}$ has been chosen as the second test compound since it has been extensively studied in the frame of topological analysis $[3,29]$. For this work the electron density has been computed with CRYSTAL98 with a simple 6-21G basis set since no absolute values were searched for. The analytical topological results have been obtained with TOPOND. Different mesh sizes have been investigated: $0.05 \AA$ and $0.025 \AA$. Only the second case is presented here since it provided well-converged integration results without treating separately valence and total densities.

\subsection{Critical Points}

Table-3 gives the characteristics of all the intra- and inter-molecular BCP's, the latter being limited to the hydrogen bonds. The position and the density at the BCP's are the same, i.e. with an agreement better than $5 \cdot 10^{-4} \AA$ and $10^{-3} \mathrm{e}^{-1} / \AA^{3}$ respectively. The biggest discrepancy among curvatures is about $2 \%$ for $\lambda_{3}$ in the case of the $\mathrm{C}=\mathrm{O}$ double 
bound. For all other curvatures the agreement is better than $0.3 \%$. The kinetic energy density $\mathrm{G}_{\mathrm{CP}}$ at the $\mathrm{BCP}$ comes from exact expression in TOPOND whereas it is derived from approximated expression derived by Abramov [30] in the case of InteGriTy. The Abramov expression is known to present severe limitation in the case of shared interactions. Its use being helpful mostly in the case of inter-molecular interactions, the $\mathrm{G}_{\mathrm{CP}}$ values are nevertheless given as an indication of its validity. In both cases, the potential energy density $\mathrm{V}_{\mathrm{CP}}$ is derived from the local expression of the Virial theorem [22]. As expected very good agreement is found for the inter-molecular $0 \cdots \mathrm{H}$ BCP's where the ratio $\left|\lambda_{1}\right| / \lambda_{3}$ is far less than one (close shell interaction) [27,30]. The same kind of observations can be made for the ring $(3,+1)$ and cage $(3,+3)$ critical points which are not reported here for clarity. It can also be noticed that all the nuclear maxima $(3,-3)$ CP's are tractable because of the use of gaussian functions in computing the density. They can be located within a $0.01 \AA$ agreement between the two methods and the CP's density discrepancy remains below $10 \%$. The CPU time is there in favour of TOPOND which uses a fully automated CP search with selection of the most appropriate method: a few seconds are needed for all the atoms whereas systematic search over the whole grid takes a few minutes.

\subsection{Integrated quantities}

As mentioned above, all the quantities integrated with the help of InteGriTy that are given here have been obtained from the total electron density. Table-4 gives the integrated volume, charge, dipolar moment and eigenvalues of the quadrupolar moment tensor (cartesian co-ordinate system) for all the atoms. A very good agreement is found. The net urea molecular charge is 0.012 electron from InteGriTy and 0.003 electron for the analytical derivation which are both good results. The dipole misalignment between 
the two methods is less than one degree, whereas it can reach 15 degrees for the quadrupolar tensor eigenvectors. Table-5 gives the molecular dipole and the corresponding crystal enhancement effect. The values are again very close to each other and compare very well with that of the literature [29]. The basin search and integration CPU time is truly in favour of InteGriTy, but taking into account the time needed to build the grid, the two methods are comparable for this small number of atoms.

\section{Conclusion}

We have shown in this paper that the use of electron densities sampled on 3D grids is a valuable tool to perform topological analysis and integration of atomic quantities. We have compared the performances of the newly developed software package InteGriTy with two other well known program that use analytical expressions of the electron density to compute the topological properties. The first of them is Newprop, which is devoted to multipolar expanded experimental densities, and the second one is TOPOND, which is used for theoretical densities and gaussian basis sets. A very good overall agreement is found between the different methods. Concerning the critical points, mesh size of about $0.05 \AA$ to $0.025 \AA$ yield excellent results for bond, ring and cage critical points for all their characteristics. On the other hand nuclear maxima can be located within $0.01 \AA$ precision. This can be helpful to study purely numerically derived densities such in the case of maximum entropy method. The integrated properties, volume, charge, dipole and quadrupole are also accurately evaluated by the grid method with a reduced CPU time. It has been shown here that reducing the mesh size down to $0.025 \AA$ enables to get accurate atomic charges from the total density. For integrated atomic charges, a better agreement is found for the comparison with TOPOND on theoretical data. This may come from both the smaller mesh size adopted 
and the smoother behaviour of the gaussian functions compared to the Slater functions (cusp condition).

\section{Acknowledgment}

The authors would like to thank Prof. M. Spackman for the kind and careful attention he put on this contribution and for his well advised comments.

\section{References}

1. R.F.W. Bader, Atoms in Molecules A Quantum Theory, Clarendon Press, Oxford 1994.

2. P.Coppens, X-Ray Charge Densities and Chemical Bonding, IUCr texts on crystallography, Oxford University Press, 1997

3. C. Gatti, V.R. Saunders and C. Roetti, Crystal field effect on the topological properties of electron density in molecular crystals: The case of urea, J. Chem. Phys. 101 (1994) p.10686.

4. T. Koritsanszky, S. Howard, T. Richter, Z. Su, P.R. Mallison and N.K. Hansen, XD computer Program Package for Multipolar Refinement and Analysis of Electron Densities from X-ray diffraction Data (1995). Program XDPRO. Free University of Berlin, Germany.

5. M. Souhassou and R.H. Blessing, Topological analysis of experimental electron densities, J. Appl. Cryst. 32 (1999) p.210.

6. A. Stash and V. Tsirelson, WINPRO - A Program for Calculation of the Crystal and Molecular Properties Using the Model electron Density (2001), http://stash.chat.ru

7. R.F. Stewart and M. Spackman, VALRAY User's Manual (1983) Carnegie-Mellon University, Pittsburg, USA 
8. A. Volkov, C. Gatti, Y. Abramov and P. Coppens, Evaluation of net atomic charges and atomic and molecular electrostatic moments through topological analysis of the experimental charge density, Acta. Cryst. A56 (2000) p.252, http://harker.chem.buffalo.edu/public/topxd/.

9. M. Barzaghi, PAMoC Online User's Manual (2001), Centro del CNR per lo studio delle relazioni tra struttura e reattivita Chim, Milano, Italy. http://www.csrsrc.mi.cnr.it/barz/pamoc.

10. F.W. Biegler Koenig, R.F.W. Bader and T. Tang, AIM200, J. Compu. Chem. 3 (1982) p.317.

11. P.L.A. Popelier, MORPHY98, Comput. Phys. Comm. 93 (1996) p.212. http://morphy.uk/ch.umist.ac.uk/.

12. C. Katan, P. Rabiller, C.Lecomte, M.Guezo, V. Oison and M.Souhassou, Numerical computation of critical properties and atomic basins from three-dimensional grid electron densities, J. Appl. Cryst. 36 (2003), p.65. http://www.gmcm.univrennes1.fr/moleculaire/integrity/

13. R.F.W. Bader, Atoms in Molecules A Quantum Theory, chap. 2, Clarendon Press, Oxford 1994.

14. B. B. Iversen, F. K. Larsen, M. Souhassou and M. Takata, Experimental evidence for the existence of non-nuclear maxima in the electron-density distribution of metallic beryllium. A comparative study of the maximum entropy method and the multipole refinement method. Acta Cryst. B 51 (1995), p.580.

15. E. Nishibori, P. Rabiller, M. Christensen, G.J. Snyder and B. B. Iversen, X-ray charge density of high performance thermoelectric Zn4Sb3, in press.

16. V. Oison, C. Katan, P. Rabiller, M. Souhassou and C. Koenig, Neutral-ionic phase transition: A thorough ab initio study of TTF-CA, Phys. Rev. B67 (2003), 035120-1 
17. P.E. Blöchl, Projector augmented-wave method, Phys. Rev. B50 (1994), p.17953

18. N.K. Hansen and P. Coppens, Testing aspherical atom refinements on smallmolecule data sets, Acta. Cryst. A34 (1978) p.909

19. V. R. Saunders, R. Dovesi, C. Roetti, M.Causa, N.M. Harrison, R. Orlando and C.M. Zicovich-Wilson, CRYSTAL98 User's Manual (1998), University of Torino, Torino.

20. W.H. Press, S.A. Teukolsky, W.T. Vetterling and B.P. Flannery, Numerical Recipes, chap. 9, Cambridge University Press, 2nd ed, 1986.

21. R.F.W. Bader, T.S. Slee, D. Cremer and E. Kraka, Description of Conjugation and Hyperconjugation in Terms of Electron distribution, J. Am. Chem. Soc. 105 (1983) p.5061.

22. R.F.W. Bader, Atoms in Molecules A Quantum Theory, chap. 5, Clarendon Press, Oxford 1994.

23. C. Gatti, TOPOND98 Users Manual (1999), CNR-ISTM, Milano.

24. P.L.A. Popelier, A robust algorithm to locate automatically all types of critical points in the charge density and its Laplacian, Chem. Phys. Lett. 228 (1994) p. 160.

25. J.Cioslowski, A. Nanayakkara, A new robust algorithm for fully automated determination of attractor interaction lines in molecules, Chem. Phys. Lett. 219 (1994) p. 151.

26. W.H. Press, S.A. Teukolsky, W.T. Vetterling and B.P. Flannery, Numerical Recipes, chap. 4, Cambridge University Press, 2nd ed, 1986.

27. A. Boukhris, M. Souhassou, C. Lecomte, B. Wyncke and A. Thalal, Evolution of the structural and mean square displacement parameters in solid solutions versus concentration and temperature, J. Phys. Cond. Matter 10 (1998), p. 1621. 
28. N. Pérès, A. Boukhris, M. Souhassou, G. Gavoille and C. Lecomte, electron density in ammonium dihydrogen phosphate: non uniqueness of the multipolar model in simple inorganic structures, Acta. Cryst. A55 (1999), p. 1038.

29. M.A. Spackman, P.G. Byrom, M. Alfredsson and K. Hermansson, Influence of intermolecular interactions on multipole-refined electron densities, Acta. Cryst. A55 (1999), p. 30.

30. Y.A. Abramov, On the Possibility of Kinetic Energy Density Evaluation from the Experimental Electron-Density Distribution, Acta. Cryst. A53 (1997) p.264.

Table-1 Properties of dihydrogen phosphate intramolecular and intermolecular bond critical points. The first and the second lines correspond to InteGriTy and Newprop results respectively. The distances from $\mathrm{CP}$ to the corresponding atoms $\mathrm{A}_{1}$ and $\mathrm{A}_{2}$ are denoted $d_{1}$ and $d_{2}$ respectively. The ellipticity $\varepsilon$ is defined as $\lambda_{1} / \lambda_{2}-1$

\begin{tabular}{|c|c|c|c|c|c|c|c|c|}
\hline$A_{1}-A_{2}$ & $\begin{array}{l}\mathrm{d}_{1} \\
\AA \\
\end{array}$ & $\begin{array}{l}\mathrm{d}_{2} \\
\AA \\
\end{array}$ & $\begin{array}{r}\rho_{\mathrm{CP}} \\
\mathrm{e}^{-} / \AA^{3} \\
\end{array}$ & $\lambda_{1}$ & $\begin{array}{c}\lambda_{2} \\
\mathrm{e}^{-} / \AA^{5} \\
\end{array}$ & $\lambda_{3}$ & $\begin{array}{c}\nabla^{2} \rho_{\mathrm{CP}} \\
\mathrm{e}^{-} / \AA^{5} \\
\end{array}$ & $\varepsilon$ \\
\hline \multirow{2}{*}{$\mathrm{O}-\mathrm{P}$} & 0.886 & 0.656 & 1.93 & -17.13 & -15.85 & +18.00 & -14.98 & 0.08 \\
\hline & 0.887 & 0.655 & 1.93 & -17.14 & -15.65 & +18.11 & -14.68 & 0.09 \\
\hline \multirow{2}{*}{$\mathrm{O}-\mathrm{H}$} & 0.988 & 0.251 & 1.20 & -21.18 & -16.52 & +10.85 & -26.85 & 0.28 \\
\hline & 0.989 & 0.251 & 1.20 & -21.18 & -17.29 & +10.54 & -27.93 & 0.22 \\
\hline \multirow{2}{*}{$\mathrm{O}-\mathrm{H}$} & 0.988 & 0.251 & 1.20 & -20.51 & -17.67 & +10.90 & -27.28 & 0.16 \\
\hline & 0.989 & 0.251 & 1.20 & -21.18 & -17.29 & +10.54 & -27.93 & 0.22 \\
\hline \multirow{2}{*}{$\begin{array}{c}\mathrm{O} \cdots \mathrm{H} \\
\text { strong }\end{array}$} & 1.224 & 0.754 & 0.19 & -1.04 & -0.81 & +3.57 & +1.72 & 0.28 \\
\hline & 1.224 & 0.753 & 0.19 & -1.04 & -0.81 & +3.56 & +1.71 & 0.28 \\
\hline \multirow{2}{*}{$\begin{array}{l}\mathrm{O} \cdots \mathrm{H} \\
\text { weak }\end{array}$} & 1.575 & 1.064 & 0.04 & -0.14 & -0.12 & +0.87 & +0.61 & 0.15 \\
\hline & 1.575 & 1.063 & 0.04 & -0.14 & -0.12 & +0.87 & +0.61 & 0.15 \\
\hline
\end{tabular}


Journal of Physics and Chemistry of Solids 65 (2004) 1951-1955, doi:10.1016/j.jpcs.2004.08.006

Table-2 Integrated atomic volumes and charges in $\mathrm{NH}_{4} \mathrm{H}_{2} \mathrm{PO}_{4}$.

\begin{tabular}{ccccccccc}
\hline Atom & $\mathrm{P}$ & $\mathrm{O}$ & $\mathrm{H} P$ & $\mathrm{H}_{2} \mathrm{PO}_{4}$ & $\AA^{3} \mathrm{~N}$ & $\mathrm{H}_{\mathrm{N}}$ & $\mathrm{NH}_{4}$ & Cell \\
\hline $\begin{array}{c}\text { Volume } \\
\AA^{3}\end{array}$ & 2.19 & 18.28 & 0.40 & 76.11 & 14.52 & 3.16 & 27.15 & 413.35 \\
\hline & 2.27 & 18.28 & 0.39 & 76.19 & 14.45 & 3.15 & 27.08 & 413.06 \\
\hline Charge & +3.614 & -1.590 & +0.836 & -1.074 & -0.865 & +0.494 & +1.111 & 0.148 \\
& +3.637 & -1.596 & +0.839 & -1.069 & -0.869 & +0.496 & +1.115 & 0.184 \\
\hline
\end{tabular}

Table-3 Bond critical points in urea crystal. The second line of each row corresponds to TOPOND results.

\begin{tabular}{|c|c|c|c|c|c|c|c|c|c|c|c|}
\hline$A_{1}-A_{2}$ & $\begin{array}{l}\mathrm{d}_{1} \\
\AA \\
\end{array}$ & $\begin{array}{l}\mathrm{d}_{2} \\
\AA\end{array}$ & $\begin{array}{l}\rho_{\mathrm{CP}} \\
\mathrm{e}^{-} / \AA^{3} \\
\end{array}$ & $\lambda_{1}$ & $\begin{array}{c}\lambda_{2} \\
\mathrm{e}^{-} / \AA^{5} \\
\end{array}$ & $\lambda_{3}$ & $\begin{array}{c}\nabla^{2} \rho_{\mathrm{CP}} \\
\mathrm{e}^{-} / \AA^{5} \\
\end{array}$ & $\left|\lambda_{1}\right| / \lambda_{3}$ & $\varepsilon$ & $\begin{array}{r}\mathrm{G}_{\mathrm{CP}} \\
\mathrm{eV} / \AA^{3} \\
\end{array}$ & $\begin{array}{c}\mathrm{V}_{\mathrm{CP}} \\
\mathrm{eV} / \AA^{3}\end{array}$ \\
\hline \multirow[t]{2}{*}{$\mathrm{C}=\mathrm{O}$} & 0.4869 & 0.7741 & 2.352 & -17.46 & -17.21 & +19.39 & -15.28 & 0.90 & 0.014 & 71.5 & -172.3 \\
\hline & 0.4874 & 0.7736 & 2.352 & -17.49 & -17.25 & +19.13 & -15.62 & 0.91 & 0.014 & 51.1 & -131.8 \\
\hline \multirow{2}{*}{$\mathrm{C}=\mathrm{N}$} & 0.5415 & 0.8031 & 2.015 & -14.58 & -12.74 & +10.46 & -16.86 & 1.39 & 0.145 & 48.9 & -129.9 \\
\hline & 0.5415 & 0.8031 & 2.014 & -14.59 & -12.74 & +10.46 & -16.86 & 1.39 & 0.145 & 29.2 & -90.6 \\
\hline \multirow{2}{*}{$\mathrm{N}-\mathrm{H}$} & 0.7446 & 0.2648 & 2.069 & -25.99 & -24.57 & +24.13 & -26.44 & 1.08 & 0.058 & 39.9 & -130.2 \\
\hline & 0.7449 & 0.2645 & 2.069 & -26.00 & -24.59 & +24.57 & -26.52 & 1.06 & 0.057 & 9.7 & -69.9 \\
\hline \multirow{2}{*}{$\mathrm{N}-\mathrm{H}$} & 0.7386 & 0.2664 & 2.091 & -26.06 & -24.63 & +24.32 & -26.37 & 1.07 & 0.058 & 41.3 & -132.8 \\
\hline & 0.7386 & 0.2664 & 2.091 & -26.17 & -24.75 & +25.06 & -26.51 & 1.04 & 0.058 & 9.9 & -70.4 \\
\hline \multirow[b]{2}{*}{$\mathrm{O} \cdots \mathrm{H}$} & 1.2570 & 0.7353 & 0.150 & -0.92 & -0.88 & +4.55 & -2.74 & 0.20 & 0.039 & 4.4 & -3.6 \\
\hline & 1.2568 & 0.7356 & 0.155 & -0.92 & -0.88 & +4.55 & +2.74 & 0.20 & 0.039 & 4.6 & -4.1 \\
\hline \multirow[b]{2}{*}{$\mathrm{O} \cdots \mathrm{H}$} & 1.2734 & 0.7853 & 0.116 & -0.69 & -0.68 & +4.03 & +2.65 & 0.17 & 0.015 & 4.0 & -2.9 \\
\hline & 1.2733 & 0.7854 & 0.117 & -0.69 & -0.68 & +4.02 & +2.65 & 0.17 & 0.014 & 4.1 & -3.1 \\
\hline
\end{tabular}


Journal of Physics and Chemistry of Solids 65 (2004) 1951-1955, doi:10.1016/j.jpcs.2004.08.006

Table-4 Integrated atomic quantities of the urea compound. The second line of each row correspo results. $\mathrm{Q}_{1}, \mathrm{Q}_{2}$ and $\mathrm{Q}_{3}$ are the eigenvalues of the quadrupolar moment tensor.

\begin{tabular}{ccccccc}
\hline Atom & $\begin{array}{c}\text { Volume } \\
\AA^{3}\end{array}$ & $\begin{array}{c}\text { Charge } \\
\mathrm{e}^{-}\end{array}$ & $\begin{array}{c}\text { Dipole } \\
\mathrm{e}^{-} \cdot \AA\end{array}$ & \multicolumn{3}{c}{$\begin{array}{c}\text { Quadrupole } \\
\mathrm{e}^{-} \cdot \AA^{2}\end{array}$} \\
\hline $\mathrm{C}$ & 5.26 & 1.673 & 0.075 & -0.441 & +0.199 & +0.241 \\
& 5.20 & 1.676 & 0.072 & -0.437 & +0.182 & +0.255 \\
\hline $\mathrm{O}$ & 14.59 & -1.134 & 0.044 & -0.156 & +0.023 & +0.175 \\
& 14.91 & -1.129 & 0.046 & -0.171 & +0.040 & +0.211 \\
\hline $\mathrm{N}$ & 17.83 & -1.136 & 0.012 & -0.521 & +0.158 & +0.365 \\
& 17.61 & -1.135 & 0.011 & -0.546 & +0.225 & +0.321 \\
\hline $\mathrm{H}$ & 4.27 & 0.433 & 0.071 & -0.027 & -0.021 & +0.048 \\
& 4.26 & 0.433 & 0.071 & -0.027 & -0.022 & +0.049 \\
\hline $\mathrm{H}$ & 4.13 & 0.427 & 0.072 & -0.023 & -0.022 & +0.038 \\
& 4.00 & 0.427 & 0.072 & -0.022 & -0.019 & +0.041 \\
\hline
\end{tabular}

Table-5 Molecular dipole in urea crystal (Debye units).

\begin{tabular}{ccc}
\hline Method & Net dipole & Crystal effect \\
\hline InteGriTY & 7.19 & 1.62 \\
TOPOND & 7.03 & 1.89 \\
Ref. [29] & 6.68 & 1.41 \\
\hline
\end{tabular}

